\section{Cellules souches neurales et signalisation Notch}

\author{
Elisabeth Traiffort ${ }^{1}$, Julien Ferent ${ }^{2}$
}

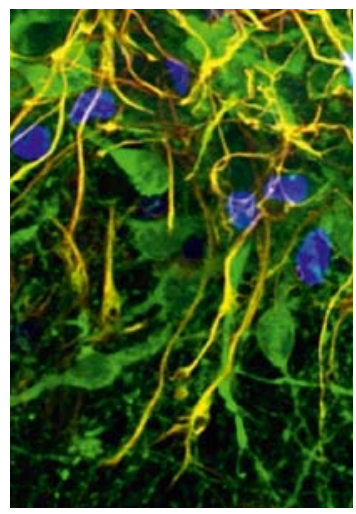

${ }^{1}$ Inserm, Univ. Paris Sud, Université Paris-Saclay, laboratoire petites molécules de neuroprotection, neurorégénération et remyélinisation, U1195, 80, rue du Général Leclerc, F-94276 Le Kremlin-Bicêtre, France;

${ }^{2}$ Institut de recherches cliniques de Montréal (IRCM), biologie moléculaire du développement neural, 110 Pine Avenue West, Montréal, Québec H2W 1R7, Canada.

elle participe à la résistance des cellules cancéreuses aux agents elisabeth.traiffort@inserm.fr sous-ventriculaire des ventricules latéraux et la zone sous-granulaire de l'hippocampe, où elle maintient la quiescence des CSN adultes, contribue au caractère hétérogène de ces cellules et exerce des effets pléiotropes au cours de la régénération du tissu neural lésé. <

Conservée durant l'évolution des espèces, la signalisation Notch est essentielle au cours du développement des invertébrés et des vertébrés. Sa capacité à contrôler les interactions cellule-cellule ainsi que la prolifération, la différenciation ou l'apoptose de divers types cellulaires lui confère un rôle majeur dans la genèse de nombreuses cellules notamment nerveuses, musculaires, sanguines, vasculaires, cardiaques, intestinales ou pancréatiques. En dehors de son rôle durant le développement, la signalisation Notch participe à l'homéostasie cellulaire dans la plupart des tissus. Ainsi, son activation favorise la synthèse du glucose et des lipides dans le foie, induit une résistance à l'insuline dans le tissu adipeux ou la polarisation des macrophages de type pro-inflammatoire dans le système immunitaire. Elle renforce aussi la quiescence des cellules souches adultes dans le muscle squelettique et le système nerveux. Enfin, induite dans la majorité des tumeurs, cation de la signalisation Notch dans la régulation des cellules souches neurales (CSN) durant le développement et chez l'adulte, et considérerons son activité dans un contexte physiologique ou pathologique.

Au cours du développement embryonnaire, les CSN sont capables de s'auto-renouveler et de générer les lignages conduisant à la formation des neurones, astrocytes et oligodendrocytes. II y a quelques décennies, la persistance de CSN dans des régions bien définies du système nerveux central (SNC) adulte fut démontrée et suscita beaucoup d'intérêt car elle offrait des perspectives thérapeutiques prometteuses pour lutter contre les processus dégénératifs du cerveau et de la moelle épinière. La connaissance approfondie des mécanismes moléculaires qui contrôlent la genèse, le maintien et la détermination des CSN est par conséquent d'une importance majeure. Parmi ces mécanismes, on trouve les signalisations Bmp (bone morphogenetic proteins), Wnt, Shh (sonic hedgehog) et Notch [46] $(\rightarrow)$. La signalisation Bmp possède

$(\rightarrow)$ Voir la Nouvelle de D. Angonin et al., $\mathrm{m} / \mathrm{s}$ $n^{\circ} 11$, novembre 2015, page 968 une activité mitogène sur les CSN embryonnaires, mais favorise la quiescence des CSN adultes. La signalisation Wnt, de son côté, induit une activité proliférative sur ces deux types cellulaires. Le morphogène Shh est impliqué dans la mise en place des différents domaines où se localisent les cellules progénitrices dans le système nerveux en développement, puis dans l'établissement et le maintien de ceux où persistent les CSN post-natales et adultes. Comme illustré dans cette revue, la voie Notch orchestre dans le temps la production des neurones et des cellules gliales, et maintient l'état indifférencié des CSN embryonnaires et adultes. 


\section{Développement et réparation du tissu nerveux}

Durant les étapes les plus précoces du développement cérébral, les CSN, appelées à ce stade cellules neuroépithéliales, subissent une série de divisions symétriques (chaque division produisant deux cellules filles identiques) dans la zone ventriculaire. Les cellules neuroépithéliales se différencient ensuite en cellules de la glie radiale qui se divisent de façon asymétrique en générant une cellule de la glie radiale et, soit un neurone immature, soit un progéniteur neural intermédiaire (PNI) de type neuronal ou oligodendrocytaire. À la fin de la période embryonnaire, la plupart des cellules de la glie radiale se différencient en astrocytes. Les cellules indifférenciées continuent à produire des PNI ou se convertissent en cellules épendymaires ou en CSN adultes [1].

Dans le cerveau adulte, deux principales régions contiennent des CSN. II s'agit de la zone sous-ventriculaire (ZSV) bordant la paroi des ventricules latéraux et de la zone sous-granulaire (ZSG) du gyrus denté de l'hippocampe $[1,46](\rightarrow)$. Les CSN adultes constituent une population hétérogène de cellules partageant avec les astrocytes leurs caractéristiques ultrastructurales et antigéniques, notamment l'expression de la protéine fibrillaire acide de la glie (GFAP,

$(\rightarrow)$ Voir la Nouvelle de D. Angonin et al., $\mathrm{m} / \mathrm{s}$ $n^{\circ} 11$, novembre 2015, page 968 glial fibrillary acidic protein). Dans la ZSV, on distingue des CSN (encore appelées cellules de type $B$ ) quiescentes ou du moins à division très lente, et des CSN activées qui expriment le récepteur du facteur de croissance épidermique (EGFR, epidermal growth factor receptor). Ces dernières se divisent activement, pour la plupart de façon symétrique, et génèrent des progéniteurs transitoires à amplification rapide (TAP, transient amplifying progenitors) aussi appelés cellules de type C [2]. La descendance des TAP est essentiellement constituée de progéniteurs neuronaux (neuroblastes ou cellules de type A) et oligodendrocytaires (OPC, oligodendrocyte progenitor cells) qui migrent respectivement dans les bulbes olfactifs et les passages de fibres proches de la ZSV. La ZSG contient, quant à elle, deux types de CSN de morphologie respectivement radiale (type 1) ou horizontale (type 2a). Ces CSN expriment des marqueurs tels que la nestine, Sox2 (sex determining region $y$-box2) et BLBP (brain lipid binding protein). Les cellules de type $2 a$ se divisent plus rapidement et se différencient en cellules de type $2 b$ qui commencent à exprimer les marqueurs de neuroblastes, puis deviennent des neurones matures dans le gyrus denté [3]. Dans des modèles d'ischémie, de démyélinisation ou de lésion corticale développés chez le rongeur, les CSN de la ZSV quittent leur état de quiescence. Les précurseurs produits sont recrutés par la lésion sous forme de neurones, oligodendrocytes ou astrocytes importants pour le processus régénératif. À l'issue de la régénération, une partie des CSN activées retourneront à l'état de quiescence $[4,5]$. L'homéostasie du tissu nerveux repose sur l'existence d'un équilibre entre le maintien des cellules souches et leur différenciation en cellules neurales matures. La signalisation Notch est un élément clé de cet équilibre.

\section{La signalisation Notch}

Initialement identifiée chez la drosophile en 1914, la signalisation Notch doit son nom à l'existence de petites encoches présentes sur les ailes de la mouche mutante. Cette voie permet une communication à courte distance entre cellules proches. Les signaux Notch interviennent lorsqu'une cellule doit faire un choix entre des programmes développementaux préexistants. Alors qu'un seul récepteur Notch et deux ligands (Delta et Serrate) sont présents chez la drosophile, quatre récepteurs (Notchl-4) et plusieurs ligands dits canoniques, Delta-like (DIII, DII3, DII4) et Jagged (Jagl, Jag2), existent chez les mammifères [6, 7]. Ligands et récepteurs sont des protéines transmembranaires de type I, exprimées par des cellules voisines (Figure 1). Ils possèdent dans leur domaine extracellulaire des motifs \&GF (epidermal growth factor) au niveau desquels s'établissent les interactions. D'autres ligands dits non canoniques ont été identifiés, notamment dans le SNC des vertébrés [8], parmi eux F3/contactine [9] ou DNER (delta/notch-like epidermal growth factor-related receptor) [10].

Deux types d'interactions ligands-récepteurs ont été décrits et modélisés [11]. Le ligand exprimé par une cellule peut lier le récepteur présenté par une cellule voisine. On parle alors d'interaction en trans qui conduit à l'activation du récepteur (ou transactivation). Alternativement, l'interaction peut avoir lieu entre ligand et récepteur exprimés par la même cellule. Cette interaction dite en cis ne déclenche aucun signal. On parle dans ce cas de cisinhibition car la cellule présente alors une capacité réduite à détecter les signaux envoyés par une cellule voisine (Figure 2A).

L'activation du récepteur Notch par les ligands canoniques se traduit par une séquence d'événements protéolytiques médiés par les protéines ADAM ( $a$ disintegrin and metalloproteinase) et $\gamma$-sécrétase. À l'issue de cette séquence, le domaine intracellulaire de Notch (NICD, Notch intracellular domain) est transloqué dans le noyau de la cellule réceptrice. En présence des protéines de type Mastermind (MAML, Mam-like proteins), NICD forme un complexe avec la protéine liant I'ADN RBPj (recombination signal binding protein for immunoglobulin kappa $j$ region), tandis que le domaine extracellulaire du récepteur est endocytosé avec le ligand dans la cellule exprimant ce dernier. Le complexe NICD-RBPj active la transcription des gènes cibles de la voie, en particulier les gènes Hes (hairy and enhancer of split) et les gènes associés Hey (hairy and enhancer of splitrelated with $Y R P W$ motif), qui appartiennent à une famille de répresseurs transcriptionnels de la famille bHLH (basic helix-loop-helix). Les protéines Hes/ Hey inhibent notamment la transcription des gènes proneuraux tels que Mashl dans la partie ventrale du cerveau antérieur, et Neurogenin 2 (Ngn2) dans le 


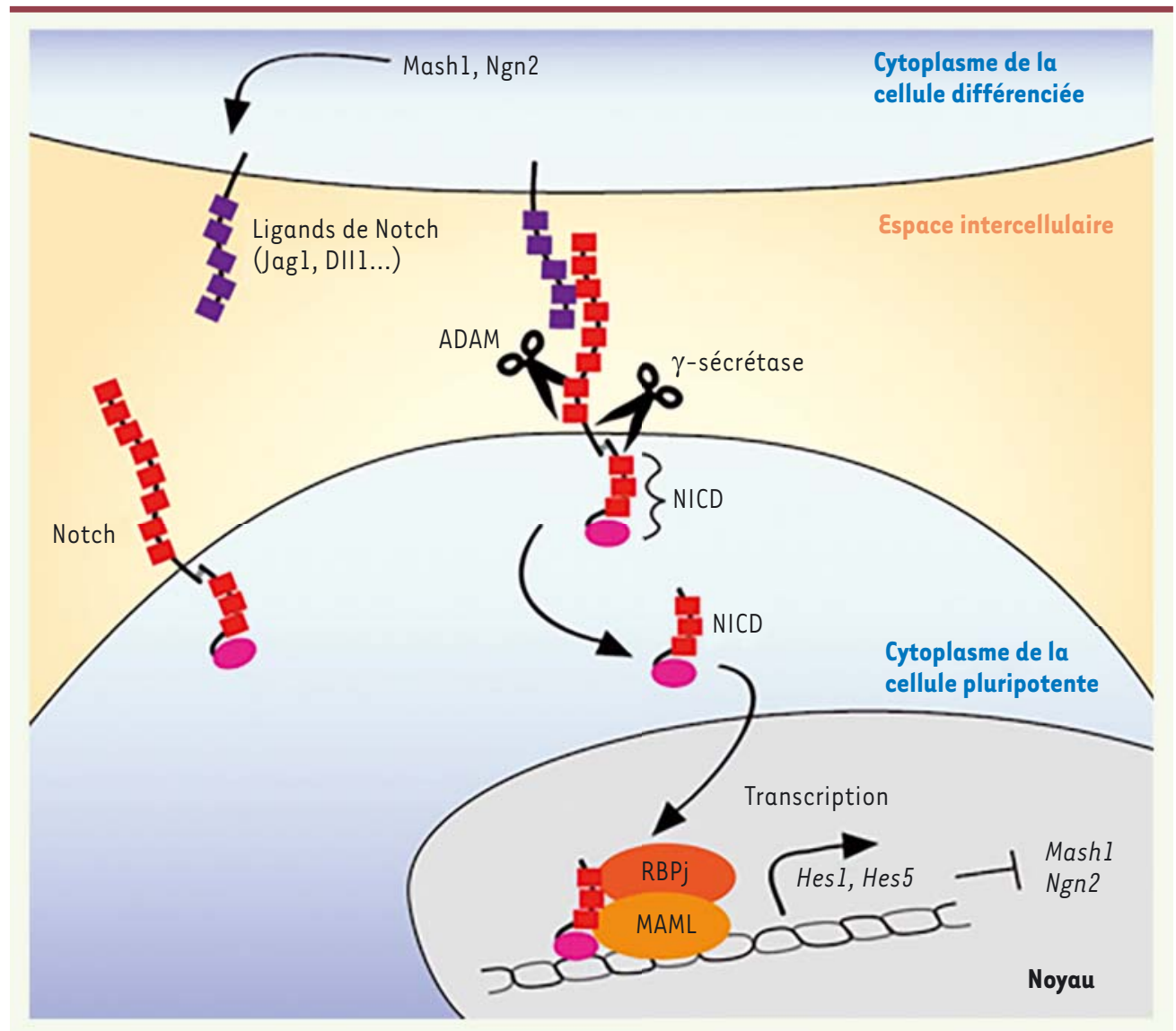

Figure 1. La voie de signalisation Notch. Les protéines pro-neurales Mashl et Neurogénine 2 (Ngn2), exprimées par la cellule différenciée, induisent l'expression des ligands de Notch qui activent une cascade intracellulaire dans la cellule voisine. L'activation du récepteur par le ligand met en jeu une série de clivages protéolytiques qui débute par une métalloprotéase de type ADAM ( $a$ disintegrin and metalloproteinase) et se termine par le complexe $\gamma$-sécrétaseprésénilline. Ces clivages résultent en la libération du domaine intracellulaire de Notch (NICD, Notch intracellular domain) qui, après translocation dans le noyau, agit en conjonction avec un complexe formé de RBPj (recombination signal binding protein for immunoglo-

bulin kappa j region) et des molécules de type Mastermind (MAML) pour induire l'expression de Hes I (hairy and enhancer of split 1) et Hes5. Les facteurs de transcription Hesl/5 répriment l'expression des gènes pro-neuraux maintenant la cellule à l'état de cellule pluripotente.

néocortex, ce qui empêche les cellules progénitrices indifférenciées de s'acheminer vers le processus de différenciation neuronale. Les cellules exprimant un ligand de la signalisation Notch se trouvent donc engagées dans une voie de différenciation tout en maintenant leurs voisines dans un état non différencié via l'activation du récepteur Notch présent sur ces dernières. Ce processus porte le nom d'inhibition latérale (Figure 1). II nécessite un contrôle étroit assuré par de nombreuses protéines telles que Botch, qui interfère avec la protéolyse et le trafic de Notch [12], les protéines Fezf [13] ou l'oncogène Bcl6 (B-cell lymphoma 6) [14] qui sont impliquées dans la répression des gènes de la famille Hes.

\section{Notch dans le système nerveux en développement}

Comprendre le rôle physiologique de la signalisation Notch a nécessité le développement de plusieurs modèles génétiques utilisés comme rapporteurs de son activité. Différentes associations de promoteurs (RBPj, Hesl, Hes5) et de rapporteurs (EGFP [enhanced green fluorescent protein], LacZ [ $\beta$-galactosidase], EYFP [enhanced yellow fluorescent protein], Venus) ont été réalisées. Les principaux outils génétiques développés et les principales avancées liées à leur utilisation sont présentés dans le Tableau I.
Rôle de Notch dans la différenciation neuronale et l'équilibre entre CSN et progéniteurs

Au cours des phases précoces du développement, la signalisation Notch est impliquée dans le processus de sélection des progéniteurs neuraux. Ainsi, au sein d'une population de progéniteurs communiquant via Notch, seules les cellules régulant négativement cette signalisation deviennent des progéniteurs neuraux, alors que les autres s'orientent vers un destin de cellule épidermique chez la drosophile [15], ou participent au maintien du stock de CSN chez les vertébrés. En accord avec cette activité, la perte du facteur de transcription Hesl, comme l'invalidation de Notchl ou de RBPj, se traduisent par la diminution du nombre de CSN et l'augmentation prématurée et excessive du nombre de progéniteurs neuraux intermédiaires (PNI) chez les vertébrés $[6,7]$.

Comme précédemment mentionné, cette activité de la voie Notch porte le nom d'inhibition latérale, plus généralement définie comme un processus par lequel des cellules adjacentes équipotentes établissent une différence d'identité par échange réciproque de signaux 


\begin{tabular}{|c|c|c|}
\hline Lignée murine & Principale avancée liée à l’utilisation de la lignée & Référence \\
\hline pRBPj-દGFP & $\begin{array}{l}\text { Démonstration de la capacité de réponse des CSN et des PNI à l'activation du récepteur } \\
\text { Notch avec transcription des facteurs Hes uniquement dans les CSN du télencéphale en } \\
\text { développement }\end{array}$ & {$[17]$} \\
\hline pHes5-nlsLacZ & $\begin{array}{l}\text { Développement d'un outil potentiellement intéressant pour l'identification et l'isolement } \\
\text { des CSN du cerveau adulte }\end{array}$ & [8] \\
\hline pHesl-EGFP-PEST & $\begin{array}{l}\text { Visualisation du profil de transcription de Hesl dont la demi-vie de l'ordre de quelques } \\
\text { minutes nécessite l'utilisation d'un rapporteur instable, EGFP-PEST }\end{array}$ & [8] \\
\hline pHes5-EGFP-3’UTR Hes5 & $\begin{array}{l}\text { Suivi des deux populations de CSN présentes dans la ZSG adulte et caractérisation de } \\
\text { leur comportement prolifératif différentiel en présence d'un stimulus neurogénique ou au } \\
\text { cours du vieillissement }\end{array}$ & [18] \\
\hline $\begin{array}{l}\text { Notch-NLuc } \\
\text { CLuc-RBPj }\end{array}$ & $\begin{array}{l}\text { Visualisation en temps réel de l'interaction des protéines NICD et RBPj respectivement } \\
\text { fusionnées aux extrémités amino- et carboxy-terminales du rapporteur luciférase }\end{array}$ & [19] \\
\hline pHesl-Ub Luc-3'UTR Hesl & $\begin{array}{l}\text { Visualisation de l'expression oscillatoire de Hesl par le biais d'une luciférase ubiquitiny- } \\
\text { lée, Ub Luc, de demi-vie inférieure à } 10 \text { minutes }\end{array}$ & {$[8]$} \\
\hline
\end{tabular}

Tableau I. Visualisation de la signalisation Notch : les principaux outils génétiques. CSN : cellules souches neurales; EGFP : protéine fluorescente verte améliorée; Luc : luciférase; nls LacZ : gène codant la $\beta$-galactosidase fusionné à une séquence permettant son expression nucléaire; $p$ : séquence promotrice; PEST : séquence riche en proline, acide glutamique, sérine, thréonine, jouant le rôle de signal pour la dégradation des protéines; Ub Luc : gène codant la luciférase fusionné à une ubiquitine mutée conférant à la luciférase une demi-vie très courte ; 3’UTR : région régulatrice non codante située en 3 ' d'un gène ; ZSG : zone sous-granulaire.

inhibiteurs. De façon intéressante, l'inhibition latérale est étroitement associée à une caractéristique bien établie des noyaux des progéniteurs neuraux, leur capacité à se déplacer entre la position basale et apicale du neuroépithélium en fonction de l'étape du cycle cellulaire dans laquelle se trouvent les progéniteurs. Cette migration nucléaire, dite intercinétique, joue un rôle majeur dans la régulation de la neurogenèse via Notch chez le poisson zèbre, comme le suggère l'analyse du mutant Mok chez lequel les mouvements nucléaires sont interrompus tandis qu'un excès de PNI est généré aux dépens des CSN [16]. Les progéniteurs en phase de mitose possèdent un noyau en position apicale et expriment fortement le récepteur Notch. À l'inverse, les progéniteurs en phase de synthèse d'ADN, dont le noyau est localisé en position basale, expriment fortement le ligand Delta de Notch. Ainsi, toutes les cellules constituant le neuroépithélium ont une probabilité équivalente de libérer ou de recevoir un signal Notch. Cette caractéristique disparaît si les mouvements nucléaires sont interrompus (Figure 2B). Une conséquence de ce mécanisme est l'existence d'une expression oscillatoire de Hesl (dans la cellule réceptrice du signal Notch) et de DllI (dans la cellule émettrice du signal Notch), identifiée in vitro et in vivo dans le télencéphale de souris en développement, au cours de la phase neurogénique (Figure 3A). Hesl oscille avec une périodicité de 2 heures, assure sa propre régulation et réprime régulièrement l'expression non seulement de Dlll, mais aussi du gène proneural Ngn2 (Neurogenin 2) [6,7]. Tant que l'expression de Ngn2 oscille, elle active une sélection de gènes parmi lesquels Dlll, mais pas les gènes nécessaires à la différenciation neuronale qui répondent lentement à Ngn2. Lors des divisions asymétriques, la cellule fille dans laquelle l'expression de DIIl est induite acquiert l'identité de PNI, alors que l'autre conserve l'identité de CSN. DIIl active Notch présent sur les cellules environnantes dans lesquelles l'expression de Ngn2 et de Dlll est alors réprimée [17]. Lorsque l'expression de Ngn2 devient stable, elle induit la différenciation neuronale. Cependant, toutes les cellules exprimant Hes 1 ne le font pas systématiquement de façon oscillatoire. C'est le cas de la région isthmique (un centre organisateur de l'axe antéropostérieur du cerveau). L'expression non oscillatoire de Hesl, empêchant la production de neurones, a été proposée comme une caractéristique des cellules dormantes ou quiescentes [18]. Le maintien des cellules de la glie radiale $\mathrm{HESI}^{+}$et l'inhibition de la différenciation neuronale sont des activités conservées dans la neurogenèse du cortex cérébral humain, comme l'ont montré des expériences réalisées dans le système expérimental des tranches de néocortex fœtal en culture [19]. Par ailleurs, plus récemment et de façon inattendue, la signalisation Notch s'est révélée capable de contrôler le choix binaire que les cellules doivent accomplir pour s'orienter vers un type neuronal donné. Dans la moelle épinière de souris ou de poisson zèbre, Notch peut ainsi influencer la génération d'interneurones excitateurs ou inhibiteurs en fonction du contexte environnemental [20]. 


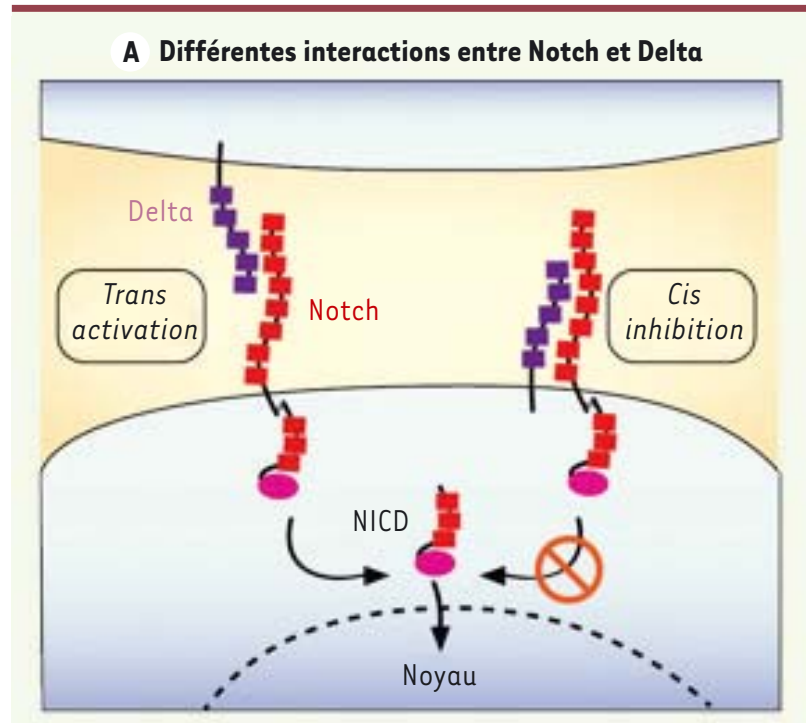

B Cycle cellulaire et mouvement nucléaire intercinétique

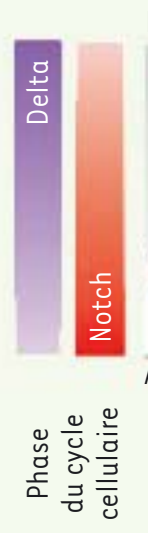
Basal

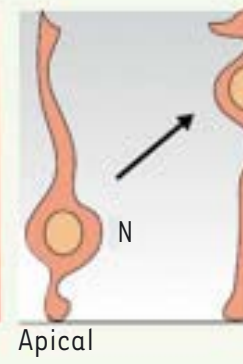

G1

$\mathrm{s}$
Figure 2. Interaction et distribution des récepteurs Notch et de leurs ligands. A. Notch et son ligand Delta peuvent interagir en trans lorsque les protéines sont exprimées par deux cellules différentes ou en cis lorsque les protéines sont exprimées par la même cellule. L'interaction en trans déclenche une activation de la signalisation Notch dans la cellule réceptrice contrairement à l'interaction en cis. B. Au cours du cycle cellulaire, les noyaux $(\mathrm{N} / 2 \mathrm{~N})$ des progéniteurs neuraux sont localisés sur le versant basal du neuroépithélium durant la phase de synthèse de l'ADN (S) et sur le versant apical pendant la phase de mitose (M). La migration nucléaire apico-basale et baso-apicale a lieu respectivement pendant la phase de croissance de la cellule (Gl) et de préparation de la mitose (G2). L'expression de Notch est plus élevée du côté apical alors que celle de Delta est plus élevée du côté basal. NICD : Notch intracellular domain.
Le rôle de la signalisation Notch dans la production des oligodendrocytes est plus complexe. La signalisation intervient dans le mécanisme qui permet aux CSN de passer de la production des neurones à celle des oligodendrocytes. Dans la moelle épinière ventrale en développement, cette transition a lieu au niveau du domaine $\mathrm{pMN}$ (progenitor domain of motoneurons) qui pro-
Rôle de Notch dans la genèse des cellules gliales

La découverte que la signalisation Notch est capable de favoriser la gliogenèse est aussi en accord avec l'idée selon laquelle Notch peut transmettre un signal instructif conduisant les cellules vers un destin spécifique. L'invalidation conditionnelle de RBPj à un stade tardif du développement embryonnaire a ainsi montré le rôle de Notch dans la différenciation des cellules astrocytaires à l'issue de la période neurogénique (Figure 3B). Les neurones en cours de différenciation secrètent les ligands qui activent Notch dans les CSN adjacentes. L'activation de Notch résulte en l'induction du facteur de transcription NFlA (nuclear factor $1 A$ ), qui permet la déméthylation du promoteur du gène codant le marqueur astrocytaire GFAP (glial fibrillary acidic protein). Le promoteur ainsi déméthylé est activé dès que les cellules reçoivent un signal activateur dérivé de la voie Jak/Stat (Janus kinase/signal transducer and activator of transcription) [21]. Plusieurs mécanismes permettent d'éviter une production excessive d'astrocytes comme la répression de Hesl par NFlA ou la dégradation du récepteur Notchl par l'ubiquitine ligase Fbxw7 en fonction du stade de développement des CSN [22]. duit successivement des motoneurones et des OPC (oligodendrocyte progenitor cells) dérivant d'un progéniteur commun exprimant le facteur de transcription Olig2 (oligodendrocyte lineage transcription factor 2). L'activation du récepteur Notch par le ligand Jag2 maintient une partie de ces progéniteurs dans un état indifférencié en inhibant la génération des motoneurones via la réduction des taux de protéine Olig2, et en prévenant la production prématurée d'OPC via le maintien d'un niveau élevé de Hes5. À l'issue de la phase neurogénique, la disparition de l'expression de Jag2 - et donc de Hes5 - annonce l'entrée du domaine pMN dans sa phase gliogénique (Figure 3C) [23]. En accord avec ces données, chez le poisson zèbre, les signaux Notch participent à l'augmentation du compartiment des précurseurs 0lig2+ qui donneront des $\mathrm{OPC}$ après blocage de la formation des motoneurones, par un mécanisme dépendant de la cycline cdknlc (cyclin-dependent kinase 1c) [24]. Au-delà de cette étape précoce de 

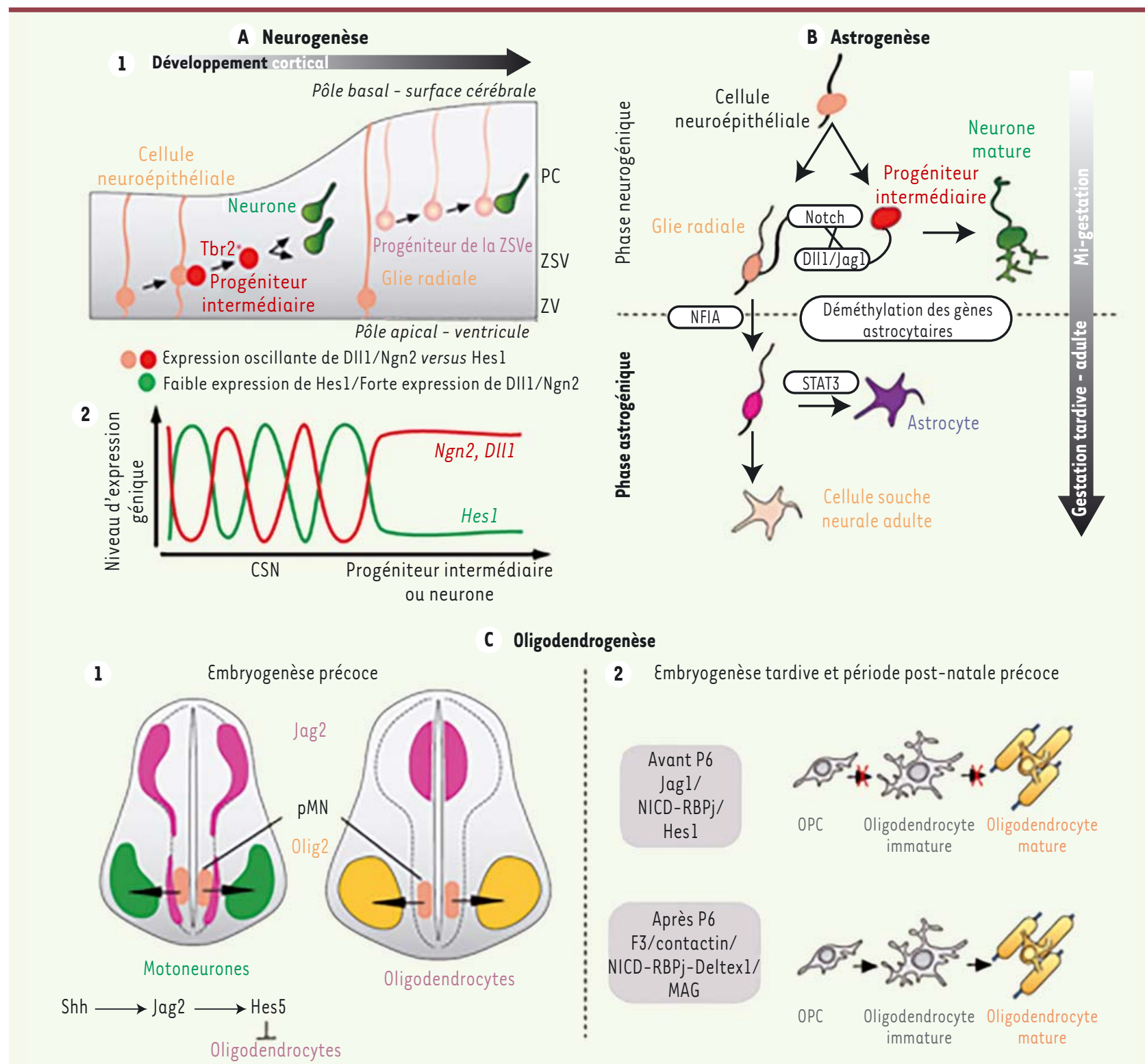

2 Embryogenèse tardive et période post-natale précoce

spécification des OPC, Jagl est exprimé par les axones des neurones jusque vers le jour post-natal 6, et active Notchl pour prévenir la maturation des OPC. Après cette période, l'expression de Jagl diminue tandis que celle du ligand non canonique F3/contactine s'accroît. L'interaction entre Notchl et F3/contactine favorise alors la différenciation des $O P C$ en induisant l'expression de protéines spécifiques de la myéline telles que MAG (myelin-associated glycoprotein) et CNPase (2', 3'-cyclic nucleotide phosphodiesterase) [9]. Il apparaît donc que les cibles transcriptionnelles de Notch varient en fonction de la nature du ligand disponible à un instant donné pour activer le récepteur.

\section{Notch dans le système nerveux adulte}

La signalisation Notch apparaît comme l'un des mécanismes essentiels de la régulation de l'équilibre entre le maintien et la diffé- renciation des CSN adultes [7, 25]. Comme chez l'embryon, les composantes de la voie, en particulier Notchl, Jagl et DIIl, sont exprimées dans les aires germinatives du cerveau adulte. Dans la zone sousventriculaire (ZSV), les ligands de Notch sont observés dans les astrocytes, dans un nombre limité de TAP (transient amplifying progenitors ou cellules $\mathrm{C}$ ), mais aussi dans les cellules épendymaires dans le cas de DIll. Notchl est, quant à lui, exprimé aux différents stades de différenciation des progéniteurs, depuis les CSN jusqu'aux neuroblastes (cellules A) [6]. Dans la zone sous-granulaire (ZSG), Jagl est détecté dans les CSN et les précurseurs neuronaux. L'expression stable ou oscillatoire des gènes Hes n'a pas encore été établie dans les CSN adultes. 
4 Figure 3. Signalisation Notch et production des neurones, astrocytes et oligodendrocytes au cours du développement embryonnaire. A-1. Dans le télencéphale embryonnaire dorsal, les cellules neuroépithéliales de la zone ventriculaire (ZV) subissent d'abord des divisions symétriques, puis se divisent de façon asymétrique pour donner des neurones ou des progéniteurs neuraux intermédiaires qui migrent respectivement dans la plaque corticale (PC) et la zone sous-ventriculaire (ZSV). Les progéniteurs neuraux intermédiaires continuent à se diviser dans la ZSV pour produire plus de neurones. Les cellules neuroépithéliales deviennent des cellules de la glie radiale dont certaines se différencient en progéniteurs de la ZSV externe (ZSVe), qui émettent un prolongement uniquement vers le versant basal. A-2. Dans les CSN (cellules souches neurales), les oscillations de Hes1 (hairy and enhancer of Split 1) contrôlent celles de Ngn2 (Neurogenin 2). Les oscillations de Dll1 (Delta-like protein 1) sont cycliquement induites par Ngn2 et réprimées par Hesl. Lorsque l'expression de Hesl disparaît, celle de Ngn2 est induite de façon continue, ce qui déclenche la différenciation neuronale. B. À mi-parcours de la gestation, les cellules neuroépithéliales se divisent de manière asymétrique et génèrent un progéniteur neural intermédiaire de type neuronal et une cellule de la glie radiale. Les progéniteurs neuraux intermédiaires, qui se différencieront ultérieurement en neurones, expriment les ligands de Notch qui vont activer les récepteurs Notch sur la glie radiale avoisinante. Cela permet l'induction de la transcription du facteur NFIA (nuclear factor IA) nécessaire à la déméthylation du promoteur des gènes spécifiques des astrocytes. Le promoteur déméthylé est alors capable de lier STAT3 (signal transducer and activator of transcription 3), qui active la transcription du gène astrocytaire. Alternativement, les cellules de la glie radiale peuvent devenir des CSN adultes de type astrocytaire. C-1. Dans la partie ventrale du tube neural, le domaine pMN (progenitor domain of motoneurons) contient des progéniteurs 0 lig2+ (rose) qui génèrent successivement des motoneurones (vert) et des oligodendrocytes (orange). Lorsqu'il est exprimé dans le domaine pMN, Jag2 maintient les progéniteurs de la partie latérale de ce domaine dans un état indifférencié, alors que les progéniteurs proches de la ligne médiane qui ne sont pas en contact avec Jag2 se différencient en motoneurones. Pendant cette phase neurogénique, Hes5 induit par Jag2, lui-même contrôlé par le morphogène Sonic Hedgehog (Shh), empêche la différenciation prématurée des progéniteurs de la partie latérale de pMN en cellules oligodendrocytaires. À l'issue de cette phase, l'absence d'expression de Jag2 dans le domaine pMN autorise l'oligodendrogenèse. C-2. Au cours de l'embryogenèse tardive jusqu'au sixième jour post-natal (P6), la voie Notch canonique est activée par le ligand Jagl et induit la transcription de Hesl qui empêche la maturation des OPC (oligodendrocyte progenitor cells). Après $\mathrm{P} 6$, la voie Notch non canonique est activée par le ligand $\mathrm{F} 3$ /contactine et induit la transcription du gène codant la glycoprotéine associée à la myéline (MAG [myelin-associated glycoprotein]) et par conséquent la différenciation des OPC.

L'inactivation conditionnelle et inductible de RBPj ou de Notchl dans les cellules neurales indifférenciées nestine ${ }^{+}$montre le rôle primordial de la signalisation Notch canonique dans le maintien à long terme des CSN adultes (cellules B) de la ZSV (Figure 4). Le phénotype global des animaux mutants reflète une conversion prématurée des CSN en TAP, cellules qui, douées d'une capacité d'autorenouvellement limitée, finiront par se différencier en neurones. Des discordances existent pourtant entre les deux phénotypes. Elles révèlent que Notchl est capable de maintenir les CSN actives en prévenant leur transition vers les TAP, mais est incapable de maintenir les CSN quiescentes, laissant supposer que des récepteurs autres que Notchl pourraient être impliqués [26]. Notch est également indispensable pour maintenir la quiescence des cellules épendymaires de la niche et prévenir leur conversion en CSN [27]. Dans le télencéphale de poisson zèbre adulte, il a été clairement établi que les CSN alternent entre quiescence et division en fonction du niveau d'activation de Notch, qu'un mécanisme d'inhibition latérale existe aussi dans ce contexte, et que des récepteurs distincts régulent de façon différentielle les sous-populations de CSN quiescentes et activées. Ainsi, Notch3 prévient l'activation et l'amplification des CSN quiescentes, alors que Notchlb empêche la différenciation des CSN activées vers le lignage neuronal [28]. Chez la souris, le ligand DIIl exprimé dans les CSN activées est lui-même directement impliqué dans le maintien des CSN de la niche puisque, lors des divisions asymétriques, une seule des deux cellules filles est capable d'exprimer DIIl et de se différencier, alors que l'autre reste indifférenciée et quiescente [29].

Le niveau d'activation de la signalisation Notch semble contribuer à I'hétérogénéité des CSN adultes. Par exemple, un nouveau type de CSN, qui exprime Hes5 et un autre gène cible de la signalisation Notch, le gène codant la protéine de liaison des acides gras cérébraux (BLBP, brain lipid-binding protein), a été récemment caractérisé dans la ZSV de la souris adulte [2]. Par ailleurs, dans le télencéphale du poisson zèbre, diverses combinaisons Her/Hes ont été identifiées dans les cellules de la glie radiale quiescente. Comment ces expressions combinatoires régulent-elles le devenir et l'activité des CSN adultes est un point qui reste à analyser. Les mécanismes moléculaires contrôlant l'activité de Notch dans la ZSV adulte commencent aussi à être décryptés. Une communication étroite existe entre les CSN et les TAP : ces dernières, via le récepteur de l'EGF (epidermal growth factor) qu'elles expriment à leur surface, régulent négativement la signalisation Notch dans les CSN en augmentant l'expression de Numb, un activateur des 83 ubiquitine ligases qui dégradent Notch [30]. Des signalisations locales, comme par exemple la voie Hedgehog, sont aussi des régulateurs des signaux Notch dans les CSN [31].

La ZSG fait appel au même mécanisme que la ZSV pour maintenir les CSN, comme l'indique le rôle essentiel de RBPj dans le maintien à long terme des CSN dans cette région (Figure 4). L'inactivation de RBPj, Notchl et Jagl est, comme précédemment, à l'origine de phénotypes divergents $[2,6,25,32]$. Ces divergences peuvent s'expliquer par l'expression de récepteurs autres que 

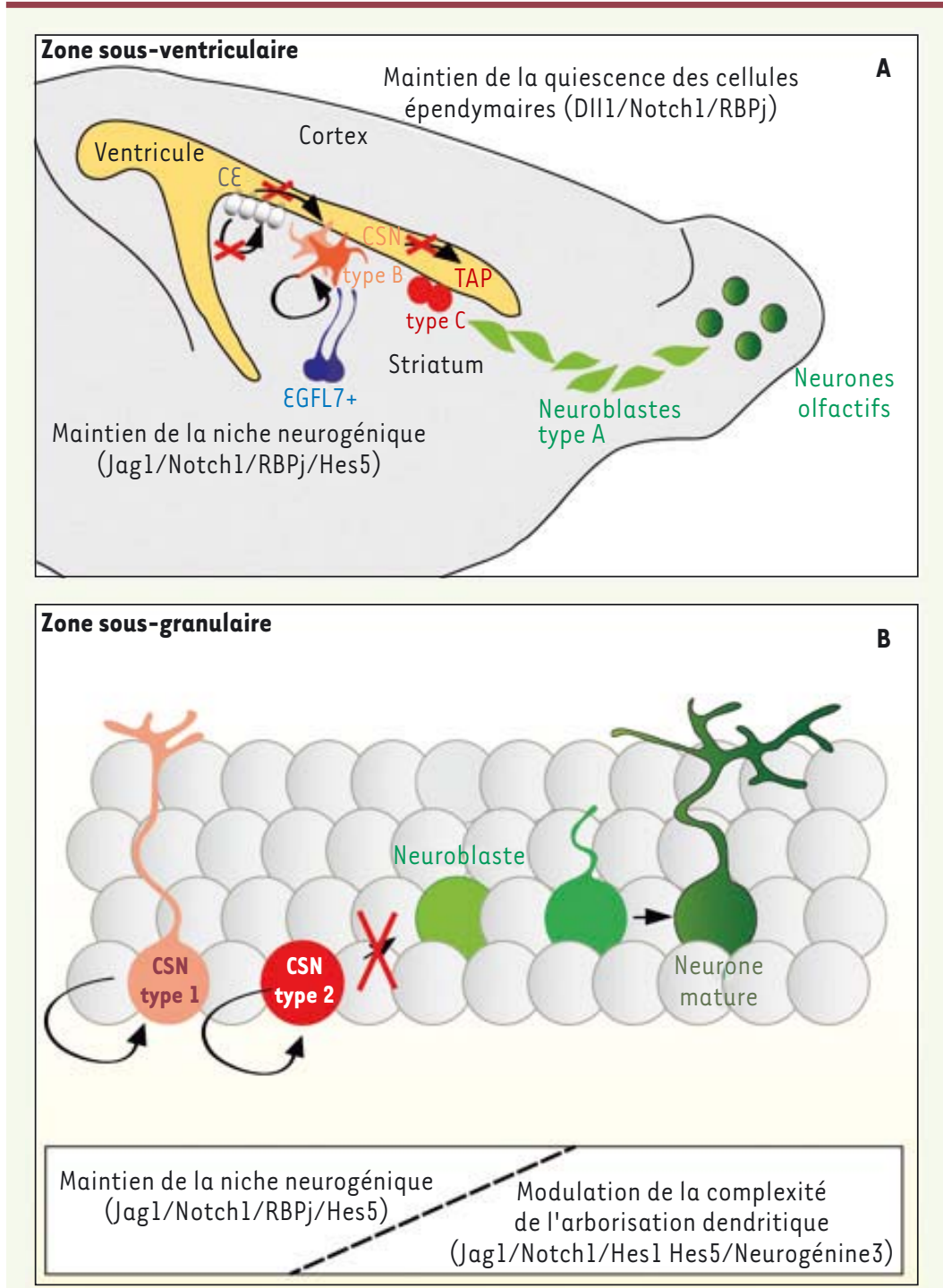

Figure 4. Notch et les aires neurogéniques adultes. A. Schéma illustrant la neurogenèse dans la zone sous-ventriculaire (ZSV) qui borde le ventricule latéral. Les cellules souches neurales (CSN) adultes (cellules $B$ ) génèrent continuellement de nouveaux neurones immatures (neuroblastes ou cellules $\mathrm{A}$ ) qui, après migration à travers le tissu cérébral, se différencient en neurones matures dans les bulbes olfactifs. Dans la ZSV, la signalisation Notch maintient les CSN, empêche leur activation et leur conversion en TAP (transient amplifying progenitors ou cellules $\mathrm{C}$ ). Elle maintient aussi les cellules épendymaires (CE) dans un état différencié prévenant leur conversion en CSN. Certains neurones striataux expriment \&GFL7 (epidermal growth factor-like domain 7), un antagoniste de Jagl. $\varepsilon n$ cas de lésion, la libération accrue de EGFL7 pourrait bloquer la signalisation Notch canonique pour favoriser la génération de quantités plus importantes de nouveaux neurones. B. Schéma illustrant la neurogenèse dans la zone sous-granulaire (ZSG). L'autorenouvellement des CSN dépend de la signalisation Notch qui par ailleurs empêche la transition CSN vers neuroblastes. La voie canonique participe également au développement de l'arborisation dendritique des neurones nouvellement générés en cours de maturation par un mécanisme faisant vraisemblablement intervenir la neurogénine 3. Les flèches noires indiquent un effet activateur de la signalisation Notch. Les flèches noires barrées d'une croix rouge indiquent un effet inhibiteur de la signalisation Notch.
Notchl dans les CSN, la mise en jeu de signalisations indépendantes de RBPj comme Jak/Stat et Hes3 [33], ou l'activation de Notch par d'autres ligands que Jagl [32]. Notch possède également un rôle additionnel dans la maturation des nouveaux neurones issus de la ZSG : il régule leur capacité à développer des neurites via le processus d'inhibition de contact $[25,34]$.

Enfin, la signalisation Notch a aussi été impliquée dans la production de deux autres catégories de neurones dans le système nerveux central post-natal. D'une part, elle maintient la prolifération et inhibe la différenciation des progéniteurs des neurones granulaires du cervelet. D'autre part, elle induit la différenciation des neurones impliqués dans le contrôle de la sensibilité nociceptive dans la moelle épinière. Ces observations permettent à l'heure actuelle de proposer qu'une activation anormale de la signalisation Notch2 pourrait participer au développement de tumeurs du cervelet (ou médulloblastomes) [35], et qu'un hypofonctionnement de Notch3 pourrait être associé à l'apparition de douleurs neuropathiques chroniques [36].

\section{Notch dans les processus régénératifs et le vieillissement du système nerveux central}

La capacité du système nerveux central à se réparer est étroitement liée à sa capacité de production de nouvelles cellules. L'implication de la signalisation Notch dans ce processus a fait l'objet de multiples études dans des contextes variables. À l'issue d'un épisode ischémique, la signalisation Notch est activée et participe au processus apoptotique, à la réponse immuno-inflammatoire et à la gliogenèse réactive contribuant à l'invasion cellulaire de la région périlésionnelle [27, 37]. L'expression de Notch] et Notch2 est notamment induite dans les neurones matures. Elle est aussi primordiale pour la prolifération des CSN de la ZSV et de la ZSG qui participent au processus de réparation. Cependant, au-delà de cette étape, une régulation négative de la signalisation Notch est ensuite nécessaire pour permettre aux nouveaux progé- 
niteurs de se différencier en neurones. L'un des modulateurs endogènes négatifs de la voie Notch pourrait être la molécule EGFL7 (epidermal growth factor-like domain 7) sécrétée à proximité de la ZSV par les neurones du striatum et capable d'entrer en compétition avec Jagl pour sa liaison à Notch dans la ZSV. En accord avec cette hypothèse, l'expression d'EGFL7 dans les CSN en culture réduit leur prolifération et oriente leur différenciation vers un excès de neurones et d'oligodendrocytes [38]. Cependant, des observations apparemment discordantes concernant le rôle de Notch dans l'ischémie cérébrale demeurent. Ainsi, l'activation de la voie s'est révélée, d'une part, améliorer les paramètres fonctionnels dans un modèle murin d'occlusion de l'artère cérébrale moyenne [33], mais, d'autre part, prévenir la mobilisation des cellules épendymaires, les empêchant de donner naissance aux neuroblastes qui contribuent à la réparation des aires endommagées [27]. Ces discordances soulignent la nécessité d'une dissection très fine des mécanismes moléculaires impliqués et de leur dynamique temporelle en réponse à l'événement lésionnel avant de pouvoir envisager la signalisation Notch comme une cible thérapeutique dans ces pathologies.

Dans le contexte de la réparation de la myéline, plusieurs études ont conclu à un effet assez modéré de la voie Notch canonique qui est un régulateur négatif de la différenciation des OPC (oligodendrocyte progenitor cells). Ce résultat est sans doute lié à l'activation compétitive de la signalisation Notch non canonique qui, à l'inverse, induit la différenciation de ces cellules [39]. L'expression élevée de Notchl par les OPC et de F3/contactine par les axones démyélinisés, dans des lésions chroniques de sclérose en plaques chez l'homme, devrait ainsi favoriser la réparation. Cependant, il est apparu que dans ces lésions, une insuffisance du transport intranucléaire du domaine intracellulaire de Notch (NICD, Notch intracellular domain) existe, lié à l'expression accrue pathologique d'un inhibiteur de cette translocation [40]. Des régulateurs positifs de la voie Notch canonique participent aussi au blocage de la réparation. C'est par exemple le cas de l'endothéline-1 qui induit le ligand Jagl dans les astrocytes réactifs présents au sein des lésions de démyélinisation [41]. Dans la maladie d'Alzheimer, l'activité Notch est réduite dans les formes familiales d'apparition précoce, mais augmentée dans les formes sporadiques comme dans d'autres pathologies neurodégénératives telles que le syndrome de Down (lié à la trisomie 21) ou les maladies à prions [37]. Les causes de telles différences dans le contrôle de la signalisation Notch restent à être élucidées de même que la contribution effective de Notch au développement de ces pathologies. La manipulation des signalisations Notch est de toute évidence une approche à considérer dans un contexte thérapeutique. II est cependant indispensable de tenir compte d'une observation récente faite chez le poisson zèbre. Des compartiments de CSN différents (cellules neuroépithéliales her4- et cellules de la glie radiale her $4^{+}$) coexistent dès le développement embryonnaire et persistent à l'âge adulte, et ils possèdent des capacités neurogéniques variables. II sera donc important d'activer les CSN ayant l'identité régionale et l'origine développementale appropriées au processus régénératif considéré [42]. De plus, il ne faut pas perdre de vue que les processus régénératifs déclinent avec l'âge. Là encore, le rôle de la signalisation Notch dans le contrôle des différentes sous-populations de CSN reste un domaine d'investigation à part entière ; il permettra notam- ment de déterminer si ce déclin reflète un épuisement des CSN actives, une augmentation des CSN quiescentes ou, plus vraisemblablement, un état de dormance des CSN qui sont cependant susceptibles d'être réactivées [2].

\section{Conclusion}

Malgré l'étendue des connaissances actuelles sur la diversité des récepteurs Notch et de leurs ligands, leurs interactions possibles, leurs localisations subcellulaires et leurs mécanismes régulateurs, il reste encore beaucoup de questions concernant la voie Notch dans les cellules souches neurales embryonnaires et adultes (voir Encadré). L'aspect dynamique de cette signalisation requiert le développement d'approches très performantes d'imagerie en temps réel à l'échelle cellulaire et subcellulaire, mais aussi la production de systèmes de modélisation dans lesquels les aspects dynamiques peuvent être testés sur des échelles de temps extrêmement courtes. Ces approches devraient participer à une meilleure compréhension de la complexité qui accompagne, en particulier, la formation du néocortex au cours du développement cérébral tant physiologique que pathologique. Améliorer nos connaissances sur le rôle exact des signalisations Notch et l'identification des médiateurs moléculaires dépendant de Notch dans les processus neuro- et gliogéniques peut ouvrir la voie au développement de nouvelles thérapies des traumatismes, des maladies neurodégénératives ou du vieillissement pathologique, comme cela a été précédemment proposé dans des pathologies atteignant d'autres tissus de l'organisme [43, 44]. En effet, en raison de sa capacité à maintenir le caractère souche des cellules cancéreuses, la signalisation Notch est une cible majeure dans de nombreuses tumeurs y compris les glioblastomes. Son blocage est actuellement obtenu par des inhibiteurs de $\gamma$-sécrétase qui sont en phase d'essai clinique comme approche antitumorale. Des molécules plus spécifiques, notamment des anticorps dirigés contre Notch1, Notch2, Notch3 ou DII4, sont aussi progressivement développées et en cours d'évaluation clinique. La cible thérapeutique que constitue la signalisation Notch, dans les pathologies neurodégénératives par exemple, bénéficiera de toute évidence des avancées actuelles en cancérologie. Enfin, le développement de petites molécules plus spécifiques que les inhibiteurs de $\gamma$-sécrétase, capables de moduler sélectivement les composantes de la signalisation Notch, canonique ou non canonique, impliquées dans le processus pathologique à considérer, et dépourvues d'effets hors-cible liés au blocage de la signalisation Notch dans les organes périphériques, fait partie des défis thérapeutiques actuels [45]. $\diamond$ 


\section{Questions ouvertes concernant la signalisation Notch}

- Quelles interactions ligands-récepteurs existent effectivement dans le tissu nerveux et quels sont leurs mécanismes régulateurs, leur dynamique et leur mode de coordination?

- Où ont lieu les interactions ligands-récepteurs dans les CSN hautement polarisées du cerveau en développement ou adulte?

- La migration nucléaire intercinétique intervient-elle dans l'activation de la signalisation Notch dans des régions autres que la rétine?

- Existe-t-il une expression oscillatoire des gènes Hes dans les CSN adultes?

- Quel est le rôle des signaux transmis par Notch dans le contrôle des différentes sous-populations de cellules souches du cerveau?

- Quelle est la contribution effective de la signalisation Notch dans le développement des pathologies neurodégénératives?

- Pourquoi la signalisation Notch est-elle régulée différemment en fonction du caractère familial ou sporadique de la maladie d'Alzheimer? - Quelles sont les cascades de signalisation moléculaires déclenchées par Notch et quelle est leur dynamique temporelle après une lésion ischémique, excitotoxique ou démyélinisante?

\section{SUMMARY}

\section{Neural stem cells and Notch signalling}

Development and repair of the nervous system are based on the existence of neural stem cells (NSCs) able to generate neurons and glial cells. Among the mechanisms that are involved in the control of embryo or adult NSCs, the Notch signalling plays a major role. In embryo, the pathway participates in the maintenance of NSCs during all steps of development of the central nervous system which starts with the production of neurons also called neurogenesis and continues with gliogenesis giving rise to astrocytes and oligodendrocytes. During the postnatal and adult period, Notch signalling is still present in the major neurogenic areas, the subventricular zone of the lateral ventricles and the subgranular zone of the hippocampus. In these regions, Notch maintains NSC quiescence, contributes to the heterogeneity of these cells and displays pleiotropic effects during the regeneration process occurring after a lesion. $\diamond$

\section{LIENS D'INTÉRÊT}

Les auteurs déclarent n'avoir aucun lien d'intérêt concernant les données publiées dans cet article.

\section{RÉFÉRENCES}

1. Kriegstein A, Alvarez-Buylla A. The glial nature of embryonic and adult neural stem cells. Annu Rev Neurosci $2009 ; 32$ : 149-84.

2. Giachino $C$, Taylor V. Notching up neural stem cell homogeneity in homeostasis and disease. Front Neurosci $2014 ; 8: 32$.

3. Ming GL, Song H. Adult neurogenesis in the mammalian brain: significant answers and significant questions. Neuron $2011 ; 70: 687-702$.

4. Benner $\varepsilon$ J, Luciano $D$, Jo R, et al. Protective astrogenesis from the SVZ niche after injury is controlled by Notch modulator Thbs4. Nature $2013 ; 497: 369-73$.

5. Lopez-Juarez A, Howard J, Ullom K, et al. Gsx2 controls region-specific activation of neural stem cells and injury-induced neurogenesis in the adult subventricular zone. Genes Dev $2013 ; 27$ 1272-87.

6. Imayoshi I, Shimojo H, Sakamoto M, et al. Genetic visualization of notch signaling in mammalian neurogenesis. Cell Mol Life Sci $2013 ; 70$ : 2045-57.
7. Pierfelice T, Alberi L, Gaiano N. Notch in the vertebrate nervous system: an old dog with new tricks. Neuron $2011 ; 69: 840-55$.

8. Heitzler P. Biodiversity and noncanonical Notch signaling. Curr Top Dev Biol $2010 ; 92: 457-81$.

9. Hu QD, Ang BT, Karsak M, et al. F3/contactin acts as a functional ligand for Notch during oligodendrocyte maturation. Cell $2003 ; 115$ : 163-75.

10. Hsieh Fy, Ma TL, Shih Hy, et al. Dner inhibits neural progenitor proliferation and induces neuronal and glial differentiation in zebrafish. Dev Biol 2013; $375: 1-12$.

11. Sprinzak D, Lakhanpal A, Lebon L, et al. Cis-interactions between Notch and Delta generate mutually exclusive signalling states. Nature $2010 ; 465: 86-90$.

12. Chi Z, Zhang J, Tokunaga A, et al. Botch promotes neurogenesis by antagonizing Notch. Dev Cell $2012 ; 22$ : 707-20.

13. Shimizu T, Nakazawa M, Kani S, et al. Zinc finger genes Fezfl and Fezf2 control neuronal differentiation by repressing Hes 5 expression in the forebrain. Development $2010 ; 137: 1875-85$.

14. Tiberi L, van den Ameele J, Dimidschstein J, et al. BCL6 controls neurogenesis through Sirtl-dependent epigenetic repression of selective Notch targets. Nat Neurosci 2012 ; 15 : 1627-35.

15. Heitzler P, Simpson P. The choice of cell fate in the epidermis of Drosophila. Cell $1991 ; 64:$ 1083-92.

16. Del Bene F, Wehman AM, Link BA, Baier H. Regulation of neurogenesis by interkinetic nuclear migration through an apical-basal notch gradient. Cell $2008 ; 134: 1055-65$.

17. Formosa-Jordan $P$, Ibanes $M$, Ares S, Frade JM. Lateral inhibition and neurogenesis: novel aspects in motion. Int J Dev Biol $2013 ; 57: 341-50$.

18. Sang $L$, Coller HA, Roberts JM. Control of the reversibility of cellular quiescence by the transcriptional repressor HES1. Science 2008 ; 321 : 1095-100.

19. Lui JH, Hansen DV, Kriegstein AR. Development and evolution of the human neocortex. Cell $2011 ; 146: 18-36$.

20. Mizuguchi R, Kriks S, Cordes R, et al. Ascll and Gshl/2 control inhibitory and excitatory cell fate in spinal sensory interneurons. Nat Neurosci $2006 ; 9$ : 770-8.

21. Namihira M, Kohyama J, Semi K, et al. Committed neuronal precursors confer astrocytic potential on residual neural precursor cells. Dev Cell 2009; 16 : 245-55.

22. Hoeck JD, Jandke A, Blake SM, et al. Fbw7 controls neural stem cell differentiation and progenitor apoptosis via Notch and c-Jun. Nat Neurosci $2010 ; 13: 1365-72$.

23. Rabadan MA, Cayuso J, Le Dreau G, et al. Jagged2 controls the generation of motor neuron and oligodendrocyte progenitors in the ventral spinal cord. Cell Death Diff 2012 ; 19 : 209-19.

24. Park HC, Appel B. Delta-Notch signaling regulates oligodendrocyte specification. Development $2003 ; 130: 3747-55$.

25. Ables JL, Breunig JJ, Eisch AJ, Rakic P. Not(ch) just development: Notch signalling in the adult brain. Nat Rev Neurosci $2011 ; 12: 269-83$.

26. Basak 0 , Giachino C, Fiorini $\varepsilon$, et al. Neurogenic subventricular zone stem/ progenitor cells are Notchl-dependent in their active but not quiescent state.J Neurosci $2012 ; 32$ : 5654-66.

27. Carlen M, Meletis K, Goritz C, et al. Forebrain ependymal cells are Notchdependent and generate neuroblasts and astrocytes after stroke. Nat Neurosci $2009 ; 12$ : 259-67.

28. Alunni A, Krecsmarik M, Bosco A, et al. Notch3 signaling gates cell cycle entry and limits neural stem cell amplification in the adult pallium. Development $2013 ; 140: 3335-47$.

29. Kawaguchi D, Furutachi $S$, Kawai $H$, et al. DIIl maintains quiescence of adult neural stem cells and segregates asymmetrically during mitosis. Nat Commun $2013 ; 4: 1880$.

30. Aguirre A, Rubio ME, Gallo V. Notch and EGFR pathway interaction regulates neural stem cell number and self-renewal. Nature $2010 ; 467: 323-7$.

31. Ferent J, Cochard L, Faure $H$, et al. Genetic activation of Hedgehog signaling unbalances the rate of neural stem cell renewal by increasing symmetric divisions. Stem Cell Rep 2014 ; 3 : 312-23.

32. Lavado A, Oliver $G$. Jagged $l$ is necessary for postnatal and adult neurogenesis in the dentate gyrus. Dev Biol 2014 ; 388 : 11-21.

33. Androutsellis-Theotokis A, Leker RR, Soldner F, et al. Notch signalling regulates stem cell numbers in vitro and in vivo. Nature $2006 ; 442: 823-6$.

34. Sestan N, Artavanis-Tsakonas S, Rakic P. Contact-dependent inhibition of cortical neurite growth mediated by notch signaling. Science $1999 ; 286$ : 741-6.

35. Hatten ME, Roussel MF. Development and cancer of the cerebellum. Trends Neurosci $2011 ; 34$ : 134-42. 


\section{RÉFÉRENCES}

36. Rusanescu G, Mao J. Notch3 is necessary for neuronal differentiation and maturation in the adult spinal cord. J Cell Mol Med $2014 ; 18: 2103-16$.

37. Alberi L, Hoey $S \varepsilon$, Brai $\varepsilon$, et al. Notch signaling in the brain: in good and bad times. Ageing Res Rev $2013 ; 12: 801-14$.

38. Schmidt MH, Bicker F, Nikolic I, et al. Epidermal growth factor-like domain 7 (EGFL7) modulates Notch signalling and affects neural stem cell renewal. Nat Cell Biol $2009 ; 11: 873-80$.

39. Fancy SP, Chan JR, Baranzini SE, et al. Myelin regeneration: a recapitulation of development? Annu Rev Neurosci $2011 ; 34: 21-43$

40. Nakahara J, Kanekura K, Nawa M, et al. Abnormal expression of TIP30 and arrested nucleocytoplasmic transport within oligodendrocyte precursor cells in multiple sclerosis. J Clin Invest 2009; 119: 169-81.

41. Hammond TR, Gadea A, Dupree J, et al. Astrocyte-derived endothelin-1 inhibits remyelination through notch activation. Neuron $2014 ; 81: 588-602$
42. Dirian L, Galant S, Coolen M, et al. Spatial regionalization and heterochrony in the formation of adult pallial neural stem cells. Dev Cell $2014 ; 30: 123-36$.

43. Le Caignec C. Pathologies humaines et récepteurs Notch. Med Sci (Paris) $2011 ; 27: 593-5$.

44. Mayeuf $A$, Relaix F. La voie Notch : du développement à la régéneration muscle squelettique. Med Sci (Paris) $2011 ; 27: 521-6$.

45. Andersson $\varepsilon R$, Lendahl U. Therapeutic modulation of Notch signalling: are we there yet? Nat Rev Drug Discov 2014 ; 13 : 357-78.

46. Angonin $D$, Marcy $G$, Raineteau 0 . Influence des morphogènes sur la régionalisation de la zone sous-ventriculaire postnatale. Med Sci (Paris) $2015 ; 31: 968-70$

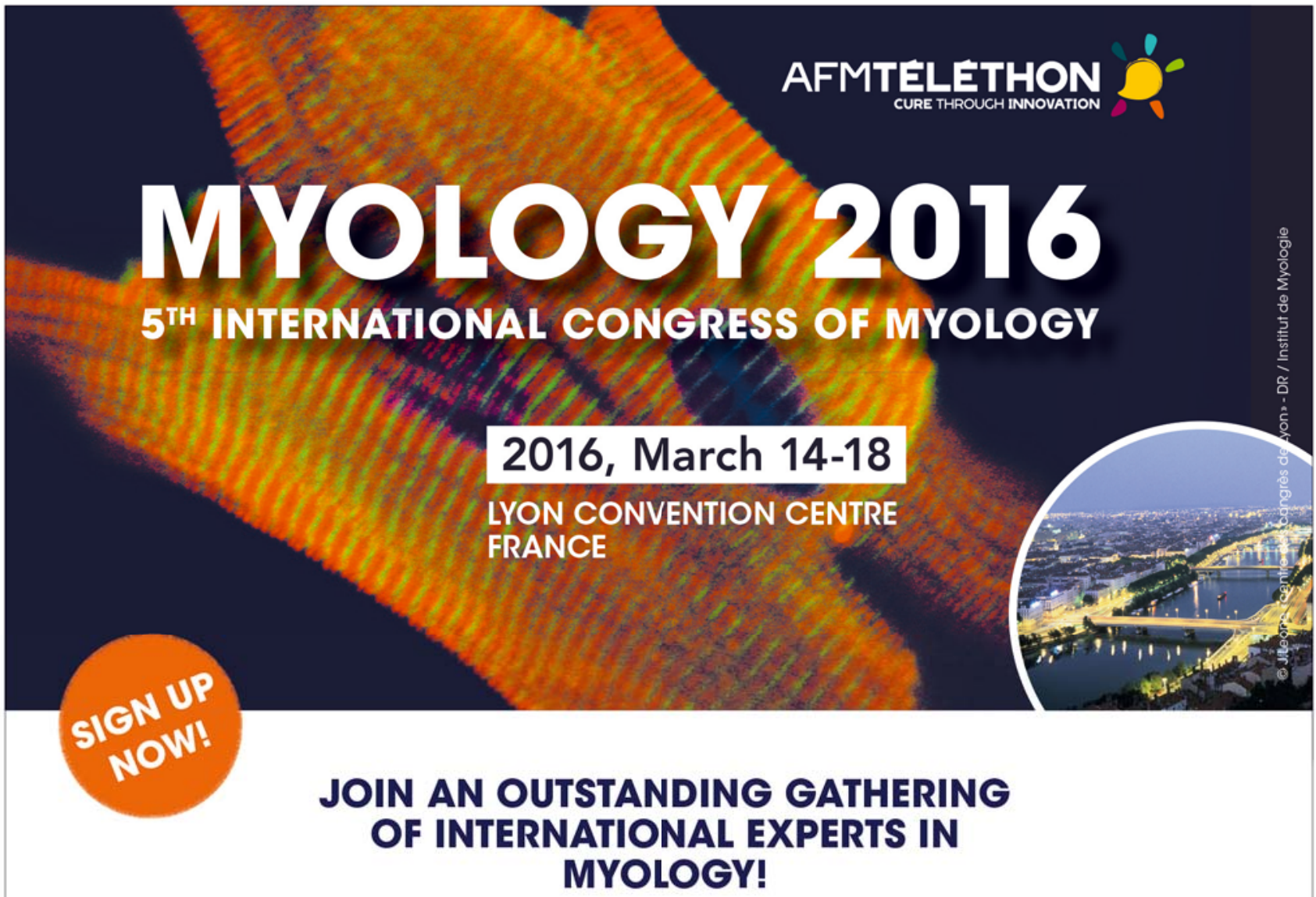

Since the last edition of our congress in 2011, Myology has undoubtedly reached a new milestone: pathophysiological and therapeutic breakthroughs multiply, and clinical trials thrive. The best specialists in this emerging discipline will come from the five continents to present and challenge their latest findings not only in fundamental research but also in clinical science and therapeutics. At Myology 2016, excellence will again be at the rendezvous!

Visit our website

www.myology2016.org

D Registration Early bird fees until January $31^{\text {th }} 2016$ 